ACL 2020

The Fourth Widening Natural Language Processing Workshop

Program of the Workshop

July 5, 2020

Seattle, USA 


\section{Platinum Sponsors}

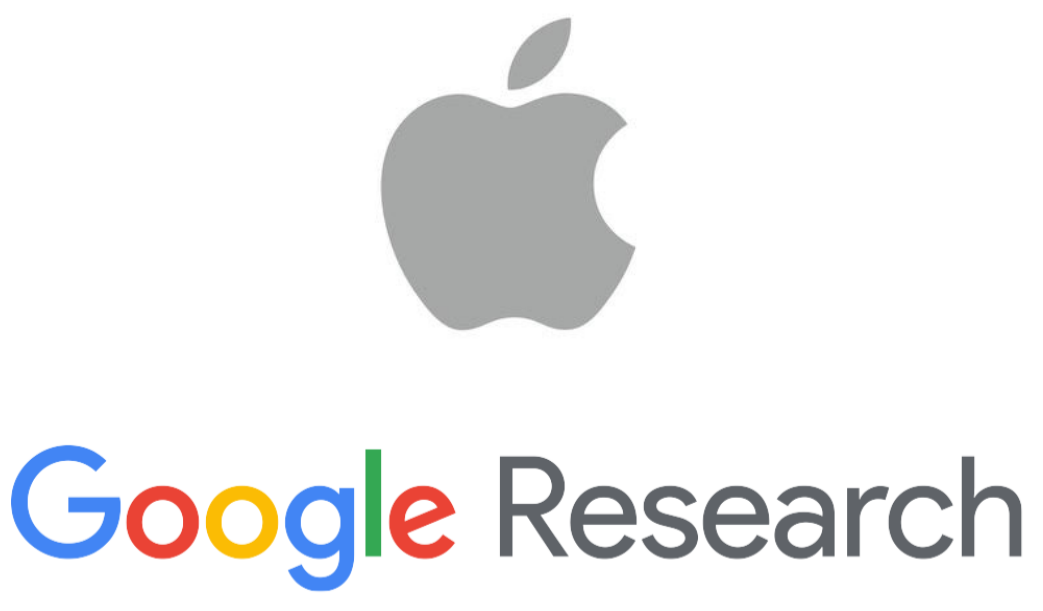

Gold Sponsors

amazon | science 


\section{Silver Sponsors}

\section{Microsoft (9) DeepMind}

\section{Bronze Sponsors}

\section{Bloomberg}

Engineering

(C) 2020 The Association for Computational Linguistics 
Order copies of this and other ACL proceedings from:

Association for Computational Linguistics (ACL)

209 N. Eighth Street

Stroudsburg, PA 18360

USA

Tel: +1-570-476-8006

Fax: +1-570-476-0860

acleaclweb.org

ISBN 978-1-952148-06-4 


\section{Introduction}

Welcome to 2020 Widening NLP Workshop!

In 2016, a select group of people met at ACL in Berlin to discuss the underrepresentation of women and other minorities in the Natural Language Processing community. The outcome of this was the first Workshop for Women and underrepresented minorities in NLP at ACL 2017: a workshop specifically dedicated to highlighting the work of groups whose work is often overlooked. We are proud to continue this work with the 2020 Widening NLP workshop.

Every year, we strive to make the workshop more inclusive. After the 2017 iteration of WiNLP, we set up two submission deadlines, one for early submissions to accommodate those who need more time for visa applications and a later deadline for those who do not have such concerns.

Following the success of the 2018 workshop, in 2019 we sought to also highlight diversity in scientific background, discipline, training, obtained degrees, and seniority. In addition, we introduced a workshopping feature, where authors themselves opted in to give feedback to their fellow authors before the papers were sent out for review.

The year 2020 brought new challenges with a global pandemic canceling all in-person conferences and workshops but has also given us the opportunity to widen our audience and authorship further. Our travel funds have been repurposed to assist with higher speed internet connections and registration fees since those are lower cost items it means we can offer assistance to even more people to join our virtual workshop.

Despite the rapid changes in 2020, we received 66 submissions! Of these submissions $54 \%$ were by women as first authors, $73 \%$ were by people of color, $54 \%$ of all papers came from outside of North America and Europe. Turning our attention to the papers which were accepted, equally encouraging numbers come up: $57 \%$ of all accepted submissions had women as their first authors, $75 \%$ of all accepted papers were by people of color, and $52 \%$ of the accepted papers came from outside of Europe and North America.

We are proud to have great invited speakers - Drs. Helena Caseli, Jose Eduardo Ochoa Luna, Verena Riesner, Rachel Tatman, as well as four excellent panelists - Drs. Anima Anandkumar, Luciana Benotti, Xanda Schofield, and MSc Jade Abbott.

We thus proudly present the 2020 Widening NLP workshop, and hope that you will enjoy the wonderful work of the authors, speakers, and panelists of this edition of the workshop.

Rossana, Samira, Erika, Ryan, Alicia, Antonios, and Khyathi. 



\section{Organizers:}

Rossana Cunha, Federal University of Minas Gerais

Samira Shaikh, University of North Carolina at Charlotte

Erika Varis, Walt Disney Studios

Ryan Georgi, University of Washington

Antonios Anastasopoulos, Carnegie Mellon University

Khyathi Raghavi Chandu, Carnegie Mellon University

Alicia Tsai, UC Berkeley

\section{Advisory Board:}

Isabelle Augenstein, University of Copenhagen

Amittai Axelrod, University of Maryland

Libby Barak, Rutgers University

Daniel Beck, University of Melbourne

Chloé Braud, CNRS - LORIA

Hal Daumé III, Associate Professor, University of Maryland

Mourad Gridach, Ibn Zohr University, Agadir

Valia Kordoni, Humboldt University Berlin

Cisca Odalipo, Kampala International University

Martha Palmer, University of Colorado

Natalie Schluter, University of Copenhagen

Tiago Torrent, Federal University of Juiz de Fora,

Aline Villavicencio, Federal University of Rio Grande do Sul, University of Essex

Hanna Wallach, Microsoft Research

Zeerak Waseem, University of Sheffield

\section{Program Committee:}

Gavin Abercrombie, University of Manchester

Fernando Alva-Manchego, University of Sheffield

Antonios Anastasopoulos, Carnegie Mellon University

Gabor Angeli, Square Inc

Miguel Ballesteros, Amazon

Libby Barak, Rutgers University

Marco Baroni, ICREA

Maria Barrett, University of Copenhagen

Chloé Braud, IRIT - CNRS

Jose Camacho-Collados, Cardiff University

Khyathi Raghavi Chandu, Carnegie Mellon University

Colin Cherry, Google

George Chrysostomou, The University of Sheffield

Kenneth Church, Baidu, USA

Marta R. Costa-jussà, Universitat Politècnica de Catalunya

Rossana Cunha, Federal University of Minas Gerais

Walter Daelemans, University of Antwerp, CLiPS

Dipanjan Das, Google AI Language

Erika Doggett, Walt Disney Studios

Luis Espinosa Anke, Cardiff University

Jennifer Foster, Dublin City University

Ryan Georgi, University of Washington 
Kevin Gimpel, Toyota Technological Institute at Chicago

Julia Hirschberg, Columbia University in the City of New York

Dirk Hovy, Bocconi University

Kristen Howell, University of Washington

Naoya Inoue, Tohoku University / RIKEN AIP

Cassandra L. Jacobs, University of Wisconsin, Madison

Huda Khayrallah, Johns Hopkins University

Kevin Knight, DiDi Labs

Rebecca Knowles, National Research Council Canada

Philipp Koehn, Johns Hopkins University

Rik Koncel-Kedziorski, University of Washington

Valia Kordoni, Humboldt-Universität zu Berlin

Ophélie Lacroix, Siteimprove

Els Lefever, LT3, Ghent University

Lucy H. Lin, University of Washington

Nitin Madnani, Educational Testing Service

Alex Marin, Microsoft Corporation

Héctor Martínez Alonso, Apple Inc

Arya D. McCarthy, Johns Hopkins University

Kourosh Meshgi, Riken AIP/ Kyoto University

Lisa Michaud, Interactions LLC

Sabrina J. Mielke, Johns Hopkins University

Aakanksha Naik, Carnegie Mellon University

Vincent $\mathrm{Ng}$, University of Texas at Dallas

Debora Nozza, Bocconi University

Eda Okur, Intel Labs

Miles Osborne, Bloomberg

Gustavo Henrique Paetzold, Federal University of Technology - Paraná

Alexis Palmer, University of North Texas

Patrick Pantel, Facebook

Yannick Parmentier, University of Lorraine

Panupong Pasupat, Google

Roma Patel, Brown University

Viviana Patti, University of Turin, Dipartimento di Informatica

Amandalynne Paullada, University of Washington

Siyao Peng, Georgetown University

Hoifung Poon, Microsoft Research

Matthew Purver, Queen Mary University of London

Dragomir Radev, Yale University

Gabriela Ramirez-de-la-Rosa, Universidad Autónoma Metropolitana

Michael Roth, University of Stuttgart

Niloofar Safi Samghabadi, University of Houston

Sashank Santhanam, University of North Carolina at Charlotte

Naomi Saphra, University of Edinburgh

David Schlangen, University of Potsdam

Alexandra Schofield, Harvey Mudd College

Brandon Sepulvado, NORC at the University of Chicago

Samira Shaikh, UNC - Charlotte

Karin Sim Smith, University of Sheffield

Noah A. Smith, University of Washington

Marco Antonio Sobrevilla Cabezudo, University of São Paulo 
Vivek Srikumar, University of Utah

Mark Steedman, University of Edinburgh

Madhumita Sushil, University of Antwerp

Jeniya Tabassum, The Ohio State University

Christoph Teichmann, Bloomberg LP

Elena Tutubalina, Kazan Federal University

Aline Villavicencio, University of Sheffield, UK

Bonnie Webber, University of Edinburgh

Wei Xu, The Ohio State University

Olga Zamaraeva, University of Washington

\section{Invited Speakers:}

Verena Rieser, Heriot-Watt University

Helena Caseli, Universidade Federal de São Carlos

José Eduardo Ochoa, Universidad Católica San Pablo

Rachael Tatman, Rasa

\section{Panelists:}

Anima Anandkumar, California Institute of Technology, NVIDIA

Jade Abbott, Retro Rabbit

Luciana Benotti, Universidad Nacional de Córdoba

Xanda Schofield, Harvey Mudd College 



\section{Workshop Program}

Sunday, July 5, 2020

8:00-9:00 Sponsored "breakfast"

9:00-10:30 Session 1: Opening, Keynote, and Posters

9:00-9:10 Opening Remarks

9:10-9:50 Keynote 1:Verena Rieser

9:50-10:30 Poster Session A

Corpus based Amharic sentiment lexicon generation

Girma Neshir Alemneh, Andreas Rauber and Solomon Atnafu

Negation handling for Amharic sentiment classification

Girma Neshir Alemneh, Andreas Rauber and Solomon Atnafu

Embedding Oriented Adaptable Semantic Annotation Framework for Amharic Web Documents

Kidane Woldemariyam and Dr. Fekade Getahun

Similarity and Farness Based Bidirectional Neural Co-Attention for Amharic Natural Language Inference

Abebawu Eshetu, Getenesh Teshome and Ribka Alemayehu

Large Vocabulary Read Speech Corpora for Four Ethiopian Languages: Amharic, Tigrigna, Oromo, and Wolaytta

Solomon Teferra Abate, Martha Yifiru Tachbelie, Michael Melese, Hafte Abera, Tewodros Gebreselassie, Wondwossen Mulugeta, Yaregal Assabie, Million Meshesha Beyene, Solomon Atinafu and Binyam Ephrem Seyoum

SIMPLEX-PB 2.0: A Reliable Dataset for Lexical Simplification in Brazilian Portuguese

Nathan Hartmann, Gustavo Henrique Paetzold and Sandra Aluísio

Token Level Identification of Multiword Expressions Using Contextual Information REYHANEH HASHEMPOUR and Aline Villavicencio 


\section{Sunday, July 5, 2020 (continued)}

Bi-directional Answer-to-Answer Co-attention for Short Answer Grading using Deep Learning

Abebawu Eshetu, Getenesh Teshome and Ribka Alemahu

Effective questions in referential visual dialogue

Mauricio Mazuecos, Alberto Testoni, Raffaella Bernardi and Luciana Benotti

A Translation-Based Approach to Morphology Learning for Low Resource Languages

Tewodros Gebreselassie, Amanuel Mersha and Michael Gasser

Monolingual corpus creation and evaluation of truly low-resource languages from Peru

Gina Bustamante and Arturo Oncevay

Tigrinya Automatic Speech recognition with Morpheme based recognition units Hafte Abera and sebsibe hailemariam

Variants of Vector Space Reductions for Predicting the Compositionality of English Noun Compounds

Pegah Alipoormolabashi and Sabine Schulte im Walde

SentiTel: TABSA for Twitter reviews on Uganda Telecoms

David Kabiito and Joyce Nakatumba Nabende

An Assessment of Language Identification Methods on Tweets and Wikipedia Articles

Pedro Vernetti and Larissa Freitas

A Comparison of Identification Methods of Brazilian Music Styles by Lyrics

Patrick Guimarães, Jader Froes, Douglas Costa and Larissa Freitas

Enabling fast and correct typing in 'Leichte Sprache' (Easy Language)

Ina Steinmetz and Karin Harbusch

AI4D - African Language Dataset Challenge

Kathleen Siminyu and Sackey Freshia

Can Wikipedia Categories Improve Masked Language Model Pretraining?

Diksha Meghwal, Katharina Kann, Iacer Calixto and Stanislaw Jastrzebski 


\section{Sunday, July 5, 2020 (continued)}

Adversarial Evaluation of BERT for Biomedical Named Entity Recognition Vladimir Araujo, Andrés Carvallo and Denis Parra

FFR v1.1: Fon-French Neural Machine Translation

Chris Chinenye Emezue and Femi Pancrace Bonaventure Dossou

Classification and Analysis of Neologisms Produced by Learners of Spanish: Effects of Proficiency and Task

Shira Wein

\section{0:30-10:50 Break (sponsored)}

\section{0:50-12:30 Session 2: Keynote and Mentoring}

10:50-11:30 Keynote 2: Helena Caseli

11:30-12:30 Mentoring and Sponsor Roundtables

\section{2:30-13:10 Lunch}

\section{3:10-15:10 Session 3: Panel, Keynote, and Posters}

13:10-13:50 Panel Discussion: Anima Anandkumar, Jade Abbott, Luciana Benotti, and Xanda Schofield

13:50-14:30 Keynote 3: José Eduardo Ochoa

14:30-15:10 Poster Session B

Developing a Monolingual Sentence Simplification Corpus for Urdu Yusra Anees, Sadaf Abdul Rauf, Nauman Iqbal and Abdul Basit Siddiqi 


\section{Sunday, July 5, 2020 (continued)}

Translating Natural Language Instructions for Behavioral Robot Navigation with a Multi-Head Attention Mechanism

Patricio Cerda-Mardini, Vladimir Araujo and Álvaro Soto

Towards Mitigating Gender Bias in a decoder-based Neural Machine Translation model by Adding Contextual Information

Christine Basta, Marta R. Costa-jussà and José A. R. Fonollosa

Predicting and Analyzing Law-Making in Kenya

Oyinlola Babafemi and Adewale Akinfaderin

Defining and Evaluating Fair Natural Language Generation

Catherine Yeo and Alyssa Chen

Political Advertising Dataset: the use case of the Polish 2020 Presidential Elections Lukasz Augustyniak, Krzysztof Rajda, Tomasz Kajdanowicz and Michał Bernaczyk

The human unlikeness of neural language models in next-word prediction

Cassandra L. Jacobs and Arya D. McCarthy

A Study on the Influence of Architecture Complexity of RNNs for Intent Classification in E-Commerce Chats in Bahasa Indonesia

Renny Pradina Kusumawardani and Muhammad Azzam

Long-Tail Predictions with Continuous-Output Language Models

Shiran Dudy and Steven Bedrick

Analyzing the Framing of 2020 Presidential Candidates in the News

Audrey Acken and Dorottya Demszky

Understanding the Impact of Experiment Design for Evaluating Dialogue System Output

Sashank Santhanam and Samira Shaikh

Studying The Effect of Emotional and Moral Language on Information Contagion during the Charlottesville Event

Khyati Mahajan and Samira Shaikh

Mapping of Narrative Text Fields To ICD-10 Codes Using Natural Language Processing and Machine Learning

Risuna Nkolele 


\section{Sunday, July 5, 2020 (continued)}

Multitask Models for Controlling the Complexity of Neural Machine Translation Sweta Agrawal and Marine Carpuat

Using Social Media For Bitcoin Day Trading Behavior Prediction Anna Paula Pawlicka Maule and Kristen Johnson

HausaMT v1.0: Towards English-Hausa Neural Machine Translation Adewale Akinfaderin

Outcomes of coming out: Analyzing stories of LGBTQ+ Krithika Ramesh and Tanvi Anand

An Evaluation of Subword Segmentation Strategies for Neural Machine Translation of Morphologically Rich Languages

Aquia Richburg, Ramy Eskander, Smaranda Muresan and Marine Carpuat

Enhanced Urdu Word Segmentation using Conditional Random Fields and Morphological Context Features

Aamir Farhan, Mashrukh Islam and Dipti Misra Sharma

Flexible Non-Autoregressive Neural Machine Translation via Repositioning Edit Operations

Weijia Xu and Marine Carpuat

\section{5:10-15:25 Break (sponsored)}


Sunday, July 5, 2020 (continued)

15:25-16:15 Session 4: Keynote and Closing

15:25-16:05 Keynote 4: Rachael Tatman

16:05-16:15 Closing Remarks 